Proceedings of the Mustansiriyah International Conference on Applied Physics (MICAP-2021)

\title{
Langmuir Probe Technique to Measure Variation of Plasma Parameters with Magnetic Field
}

\author{
S.H. ABD Muslim* \\ Department of Physics, College of Science, Mustansiriyah University, Baghdad, Iraq \\ Doi: 10.12693/APhysPolA.140.358 *e-mail: shaimaa.muslim@uomustansiriyah.edu.iq
}

\begin{abstract}
The response of a probe with varying applied potential was studied at various operating pressures and the effect of magnetic field strength on the characteristics of argon plasma was investigated. Using a single Langmuir probe, the temperature and density of electrons were measured in both the presence and absence of a magnetic field. Sheath thickness was determined and the fluctuation of the density of ion current with working pressure was also examined. The results show that the electron temperature and sheath thickness vary inversely with the gas pressure and magnetic field strength, but the values of electron density increase with increasing pressure and magnetic field. In addition to that, when a magnetic field is applied, two groups of electrons are observed. Here, the values of the ion current density in the absence of magnetic field are greater than the ion current density in the presence of an applied magnetic field.
\end{abstract}

topics: plasma diagnostics, Langmuir probe

\section{Introduction}

Over the past four decades, the use of ionic technologies for layer deposition has developed rapidly. Sputtering magnetrons are among the most used methods in this area. One major application is the deposition of functional films produced with material sputtered from cathodes [1]. When a magnetic field is applied behind the cathode, electrons move in a helical path around the magnetic field lines and traverse a significantly longer track in the plasma than in a traditional glow discharge, producing more ionizing collisions and, as a result, greater ion fluxes $[2,3]$. This provides several advantages. For instance, due to the plasma confinement close to the surface of the target, a high degree of sputtering can be achieved [4]. Reducing electron bombardment of the substrate and increasing the ionisation efficiency of the plasma gas leads to significant lowering of the voltage of the plasma and to improved pressure performance. This technique is chosen because it allows the ions to travel towards the material of the target without colliding with the region of the sheath, preserving the kinetic energy of the ions. Lower pressure means fewer collisions between neutral gas and sputtered atoms, which lowers scattering of the sputtered atom on the system's walls or its return to the surface of the target. Understanding of the plasma parameter profile will allow us to optimize the plasma chemistry, nucleation, and thin-film growth in the system [7].
There are many methods of measuring the parameters of the plasma, for example, microwave interferometry, analysis of ion energy, and electrical probe diagnostics. Electrical probes are essential diagnostic instruments in plasma research, particularly in cold plasma and at low gas pressure. These probes have the advantage of being able to perform local plasma parameter measurements $[8,9]$. The perturbation of the plasma across the position of the probe caused by the space charge sheath in front of the surface of the probe and the extraction of charged particles is detected by the immersed electrical probe. Despite the relatively simple technique, particular conditions must be satisfied in order to accurately analyze the plasma parameters derived from the probe $I-V$ characteristic [10]. These parameters are related to external parameters of the plasma system, such as the gas flow rate, dissipated power, and gas pressure.

By controlling the external parameters of the plasma system one can control the plasma parameters and consequently improve the film properties [8]. Over the last few years, extensive research has been conducted on thin film growth and characterisation. For example, Kotp et al. [11] used single Langmuir probe measurements to determine the plasma parameters $\left(T_{\mathrm{e}}, n_{\mathrm{i}}\right)$ of hollow cathode discharge of argon plasma. Chiad et. al. [12] studied the current-voltage characteristics of the Langmuir probe in the glow discharge at an operating pressure of $0.7 \mathrm{mbar}$ for three different cases without 
(a)

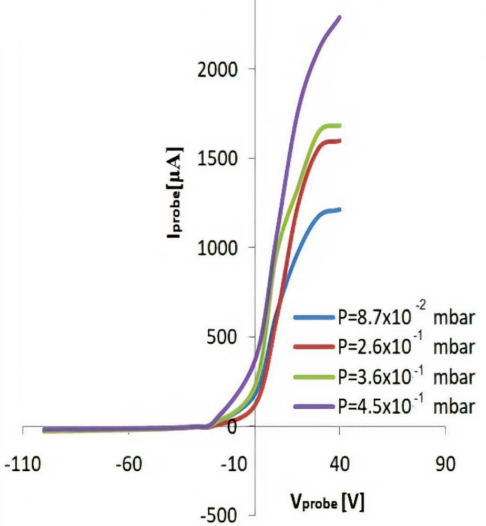

(b)

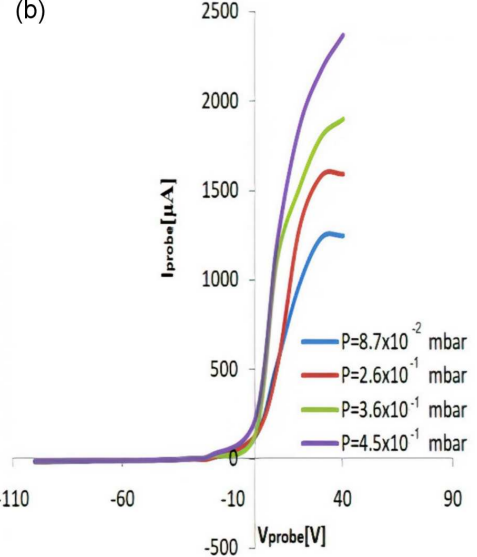

(c)

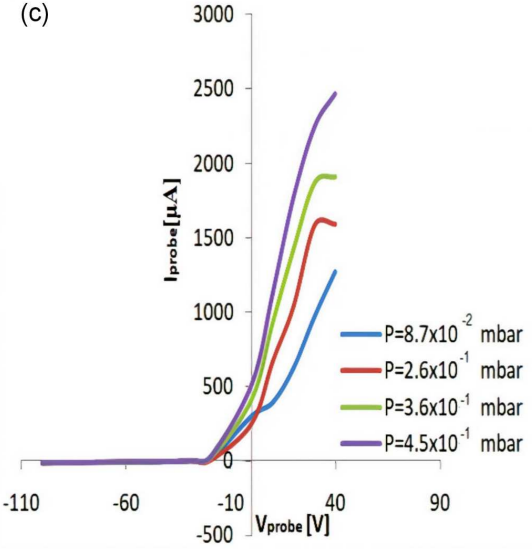

Fig. 1. The Langmuir probe voltage-current curves for various argon gas pressures: (a) $B=0$ Gauss, (b) $B=50$ Gauss and (c) $B=90$ Gauss.

magnetron, with a single magnetron, and with dual magnetrons. Ding et al. [13] utilised a combination of machine learning and Langmuir probes to determine the electron density in the positive electrode.

In the present paper, the plasma diagnostics of a magnetron is examined using Langmuir probes. The electron temperature $T_{\mathrm{e}}$, electron density $n_{\mathrm{e}}$, ion current density, and sheath thickness were calculated from the $I-V$ characteristics of the probe at various magnetic field strengths, including those in the absence of magnetic field. Measurements were conducted at different gas pressures.

\section{Materials and methods}

The experiments with use of a home-made DC magnetron sputtering apparatus were performed in a vacuum chamber made of stainless steel with a diameter of $30 \mathrm{~cm}$ and a length of $40 \mathrm{~cm}$. Two parallel circular electrodes with diameters of $10 \mathrm{~cm}$ were used, of which one (the anode) was movable and the other one (the cathode) was fixed. Both electrodes were enclosed in the vacuum chamber. The cathode magnetron assembly, which contains cooling water beneath the cathode plate, was designed and built by the researcher. The magnetic field was generated by electromagnetic coils with an outside diameter of $8.5 \mathrm{~cm}$, an inner diameter of $2.8 \mathrm{~cm}$, and a height of height of $2 \mathrm{~cm}$, allowing the magnetic field to be changed from 50 to 90 gauss when the current was changed from 0.5 to $0.75 \mathrm{~A}$. The magnetic field was measured using a Digital Gauss Meter SJ200. A rotary pump (Edward, with a flow rate of $12 \mathrm{~m}^{3} / \mathrm{h}$ ), and a diffusion pump (Alcatel, with a rate of $380 \mathrm{~L} / \mathrm{s}$ ) were used to pump the vacuum chamber. Argon gas was fed into the chamber through a needle valve and was monitored with a Pirani gauge (Edward, controller 1105). The required partial pressure was set and permitted to stabilize. Different argon pressures were used, in the range from 0.087 to 0.45 mbar. The distance between the substrate and the target was $4.5 \mathrm{~cm}$.
The glow discharge was generated by a DC power supply, and the discharge current and voltage were measured using digital multimeters. A voltage was supplied between the anode and the magnetron cathode, conserving the anode's ground. The breakdown voltage could be adjusted in a wide range of discharge values by altering the pressure. As a result of breakdown, plasma was formed between the two electrodes, causing current to flow rapidly through the system.

The tungsten probe tip was $0.5 \mathrm{~mm}$ in diameter and $2 \mathrm{~mm}$ in length. A thin glass tube isolated the wire. Variable voltage was supplied to the probe to measure the $I-V$ characteristic curves. The electron temperature $T_{\mathrm{e}}$ was calculated from the slope of the semilogarithmic plot according to [14]

$$
\text { Slope }=-\frac{E}{k_{\mathrm{B}} T_{\mathrm{e}}},
$$

where $T_{\mathrm{e}}$ and $k_{\mathrm{B}}$ represent the electron temperature $[\mathrm{K}]$ and Boltzmann's constant $[\mathrm{eV} / \mathrm{K}]$, respectively.

The electron density $n_{\mathrm{e}}$ is given by

$$
n_{\mathrm{e}}=3.73 \times 10^{13} \frac{I_{\mathrm{sat}}}{A \sqrt{k_{\mathrm{B}} T_{\mathrm{e}}}},
$$

where $I_{\text {sat }}$ is the saturation electron current, and $A$ is the probe area. The sheath width $S$ can be calculated from Child's law $[14,15]$

$$
S=\frac{\sqrt{2}}{3} \lambda_{\mathrm{D}}\left(\frac{2 e V_{0}}{k_{\mathrm{B}} T_{\mathrm{e}}}\right)^{3 / 4},
$$

where $\lambda_{\mathrm{D}}$ represents the Debye length, and $V_{0}$ is cathode potential.

\section{Results and discussion}

The voltage-current curves of the Langmuir probe are presented in Fig. 1 for argon gas pressures ranging from 0.087 to 0.45 mbar. It can be observed that the probe current increases with the increase of applied pressure. The increase of argon gas pressure leads to greater probe currents due to the growing density of neutral gas atoms, which are 

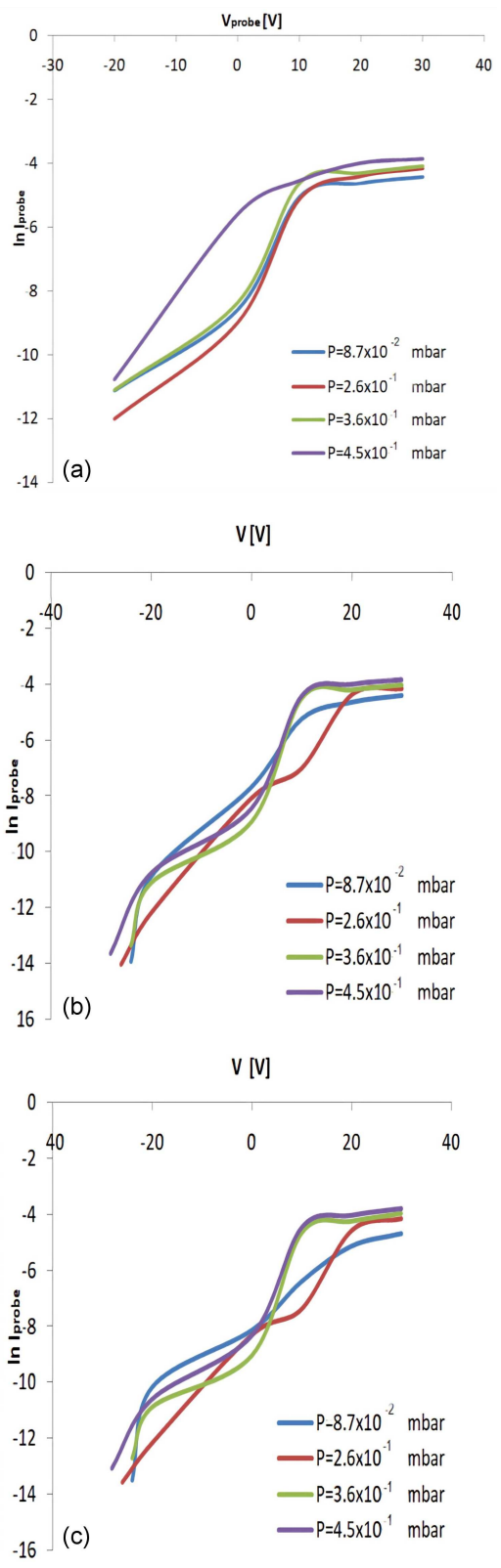

Fig. 2. Semilogarithmic plot of the probe current vs. probe voltage: (a) $B=0$ Gauss, (b) $B=50$ Gauss and (c) $B=90$ Gauss.

subject to collisions with the available charged particles. In order to calculate various plasma parameters the $I-V$ curves were divided into 3 regions, i.e., (i) the ion saturation region, where the probe current is primarily due to the attraction of positive ions to the probe, (ii) the transition region, which demonstrates that there is diffusion of electrons to the probe and where the current fluctuates exponentially with the probe voltage, and (iii) the electron saturation region, where the probe current is primarily due to the attraction of electrons to the probe.

In the electron retardation region the logarithm of the recorded probe current is plotted against probe voltage to obtain the electron temperature,

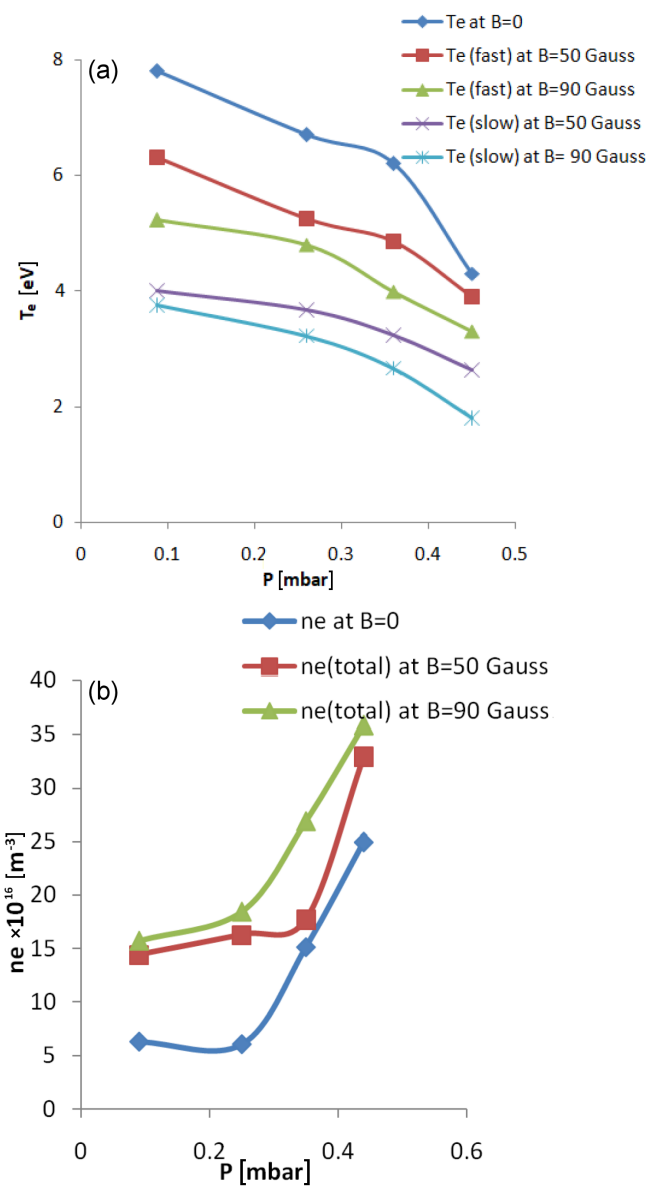

Fig. 3. (a) Electron plasma temperature $T_{\mathrm{e}}$ as a function of gas pressure in the presence and absence of magnetic field. (b) Electron density as a function of pressure in the presence and absence of magnetic field.

as shown in Fig. 2. The slope then gives the electron temperature. In Fig. 2, one can see that the electron temperature increases with increasing gas pressure, which is consistent with prior studies [16]. After application of a magnetic field, the probe signature shows the presence of two types of electrons fast and slow electrons. It is evident that the magnetic field confines the electrons to a region near the cathode, causing an increase in fast electron density, since these fast electrons would be much more energetic. Thereafter, they lose their energy through collisions, causing ionisation, and join the group of slow electrons. Subsequently, slow electrons are generated by ionization processes.

The fall in electron temperature with increasing argon pressure may be attributed to the following phenomenon when the argon pressure within the chamber raises, the number of collisions that occur between electrons and gas atoms increases, as seen in Fig. 3. As a result of the energy exchange between electrons and gas particles, the gas temperature rises, while the temperature of electrons is reduced. Also, Fig. 3a shows that in the same pressure 


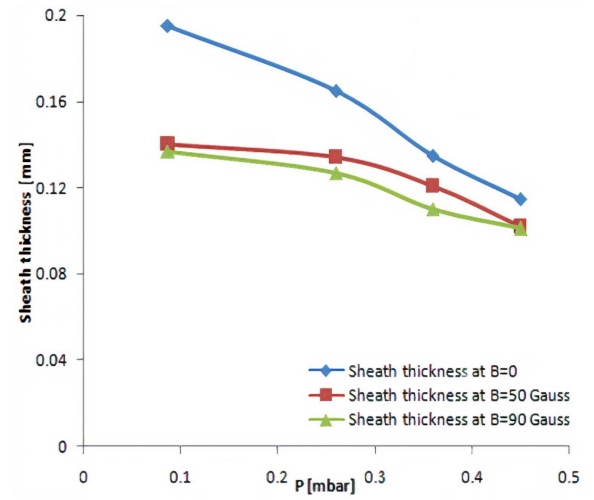

Fig. 4. The variation of sheath thickness as a function of working pressure at different magnetic field strengths.

range, the electron temperature in the presence of a magnetic field is smaller than that without a magnetic field, which is connected to the impact of magnetic fields on electrons. When the magnetic field is increased, the number of electrons confined at the cathode surface increases and the electrons lose their energy due to ionizing collisions while drifting in motion, as described by the $E \times B$ effect. This causes the electron temperature to decrease with the increase in the strength of the applied magnetic field. When the pressure and magnetic field increase the collisions between the electrons and the atoms, the ionization rate becomes higher. As a result of this higher ionization rate, the electron density at the cathode rises dramatically, as seen in Fig. 3b.

Figure 4 depicts the variation in sheath thickness $(S)$, calculated by using (3) for various magnetic fields and different pressures. The creation of a sheath is the plasma's means of maintaining net charge neutrality inside the plasma. A positively biased electrode collects the electron cloud, limiting the electric field's penetration into the plasma to a distance roughly equal to the Debye length of the electron $\lambda_{\mathrm{D}}$. When the magnetic field and pressure both increase, the cathode sheath is observed to contract. The plasma will be contained in a decreasing volume as the operating pressure rises. Also, the decrease in the sheath thickness of the cathode is due to the increase in the overall plasma density as well as the magnetic field's improved confinement of electrons at the cathode. The effect of the plasma density on the cathode sheath thickness dominates over that of the electron confinement factor.

The variation in the ion current density as a function of argon pressure is shown in Fig. 5. The ion current density in the absence of a magnetic field is higher than in its presence. At first, raising the negative bias voltage for the probe increases the observed ion current density. Since the frequency of collisions increases with higher gas pressure, the ion current density in the Ar plasma increases. Electrons collide with gas particles more often, causing
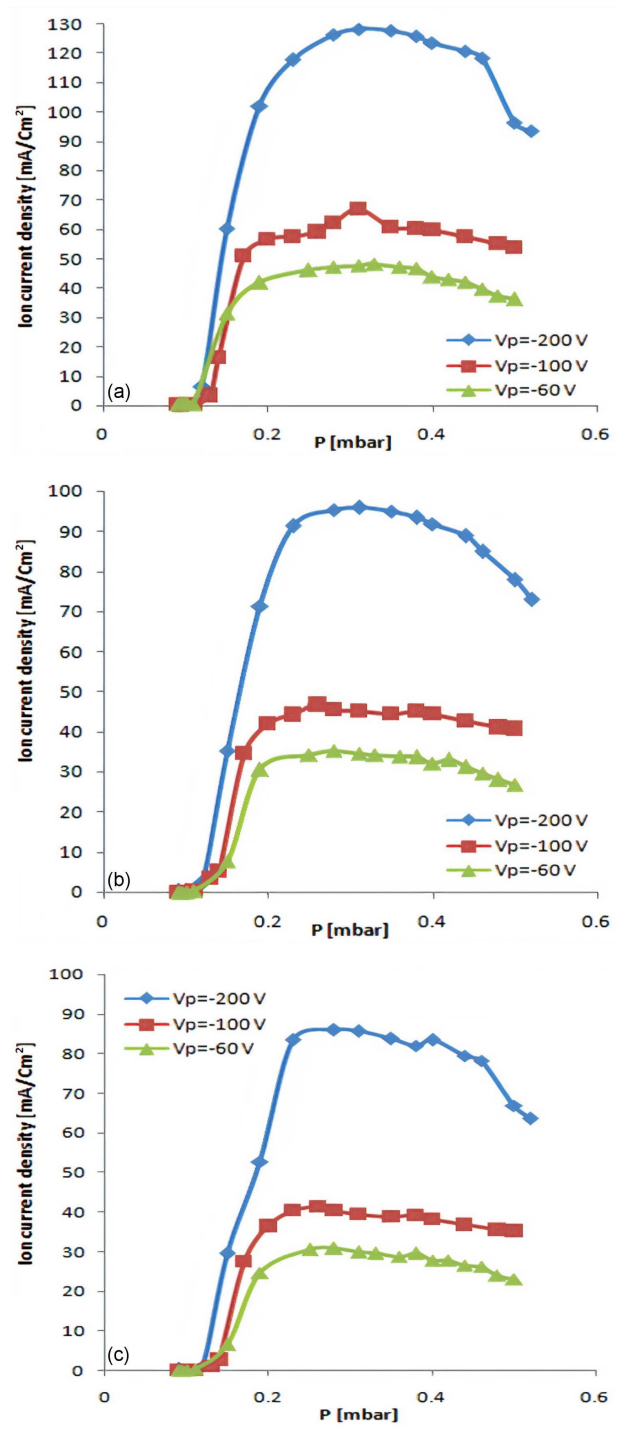

Fig. 5. The ion current density as a function of gas pressure at different applied magnetic fields and probe voltages: (a) $B=0$ Gauss, (b) $B=50$ Gauss and (c) $B=90$ Gauss.

them to ionize. Because of the enormous number of ion-atom and ion-ion collisions, the ion current density falls with increasing pressure once it achieves its maximum value. As a result, the ion scattering ratio increases.

\section{Conclusions}

In this study we observed two different electron temperatures based on the $I-V$ curves of the Langmuir probe in the presence of an applied magnetic field. The electron temperature increased when the magnetic field and argon pressure decreased, while the electron density increased when the pressure and magnetic field increased. The sheath width calculated with Child's Law, showed that the thickness increases with decreasing magnetic field and pressure. The ion current density in the presence of a magnetic field is lower than in its absence. 


\section{Acknowledgments}

The author acknowledges the Centre of Applied Physics, Ministry of Science and Technology, Baghdad, Iraq, and the Department of Physics of College of Science of Mustansiriyah University for their support in the current work.

\section{References}

[1] R. Hippler, M. Cada, Z. Hubicka, Plasma Sources Sci. Technol. 30, 045003 (2021).

[2] I.R. Agool, M.K. Khalaf, S.H. Abd Muslim, R.N. Talaq, Eng. Technol. J. 33, 1082 (2015).

[3] Y.S. Wang, W. Ding, J. Yan, Y. Wang, AIP Advances 7, 9 (2017).

[4] M.H. Suhail, F. Yousif Hadi, Indian J. Sci. Technol. 4, 1704 (2011).

[5] M.M. Abdelrahman, J. Phys. Sci. App. 5, 128 (2015).

[6] J.T. Gudmundsson, Plasma Sources Sci. Technol. 29, 113001 (2020).

[7] S. Gopikishan, I. Banerjee, S.K. Mahapatra, arXiv:1704.06503, 2017.
[8] K. Honglertkongsakul, S. Chaiyakun, N. Witit-anun, W. Kongsri, P. Limsuwan, Proc. Eng. 32, 962 (2012).

[9] D. Reiser, N. Ohno, H. Tanaka, L. Vela, Phys. Plasmas 21, 032302 (2014).

[10] S. Pfau, M. Tichy, in: Low Temperature Plasma Physics - Fundamental Aspects and Applications, Eds. R. Hippler, S. Pfau, M. Schmidt et al., Wiley-VCH, Berlin 2001, p. 131.

[11] E.F. Kotp, A.A. Al-Ojeery, Aust. J. Basic Appl. Sci. 6, 817 (2012).

[12] B.T. Chiad, O.A. Hammadi, F.J. Kadhim, M.K. Khalaf, Iraqi J. Appl. Phys. 12, 17 (2016).

[13] Z. Ding, Q. Guan, C. Yuan, Z. Zhou, Z.A Qu, AIP Advances 11, 045028 (2021).

[14] A. Heiler, R. Friedl, U. Fantz, J. Appl. Phys. 127, 113302 (2020).

[15] S.A. Irimiciuc, S. Chertopalov, J. Lancok, V. Craciun, Coatings 11(7), 762 (2021).

[16] D.B. Dreghici, B. Butoi, D. Predoi, S.L. Iconaru, O. Stoican, Polymers 12, 12 (2020). 